\title{
Embodied online dance learning objectives of CAROUSEL+
}

\author{
Kenny Mitchell* \\ Edinburgh Napier University, Roblox, 3FINERY, Cobra Simulation \\ Monica Tamariz Jessie Kennedy \\ Edinburgh Napier University, Heriot-Watt University \\ $\begin{array}{cc}\text { Elisa Mekler } & \text { Pieter van der Linden } \\ \text { Aalto University } & \text { VIVITnet }\end{array}$ \\ Edinburgh Napier University \\ Erik Herrmann \\ lain McGregor \\ Edinburgh Napier University \\ DFKI, Saarland University \\ Babis Koniaris \\ Edinburgh Napier University \\ Noshaba Cheema \\ DFKI, MPI-INF, Saarland University \\ Perttu Hämäläinen \\ Aalto University \\ Philipp Slusallek \\ DFKI, Saarland University \\ Carmen Mac Williams ${ }^{\dagger}$ \\ Grassroots Arts
}

\begin{abstract}
This is a position paper concerning the embodied dance learning objectives of the CAROUSEL $+{ }^{1}$ project, which aims to impact how online immersive technologies influence multi-user interaction and communication with a focus on dancing and learning dance together online. We aim to enable shared online social immersive experiences across the reality-virtuality continuum synchronizing audio, visual and haptic rendering. In teaching and learning to dance remotely, our models should support accessibility, style transfer and adaption of multi-modal feedback according to skills, strength, flexibility observations.
\end{abstract}

\section{INTRODUCTION}

The Internet connects individuals to the world. Phones and computers give unprecedented access not only to information but also to countless other people in the world in virtual social networks. However, a lot of online experience is passive, disconnected and disembodied. Internet users are patently part of the digital world, but often isolated from real-world sensations and feelings such as the presence of others, their touch, or their movement. We find ourselves isolated, instead of brought together, by the very technologies that are designed to connect us.

Isolation and loneliness are distressing and debilitating and have long-lasting consequences for health, including mental health, productivity, and happiness [31]. The current COVID-19 crisis and the consequent social distancing, confinement and lockdown have made the problem more acute and visible for the whole of society. The ways in which we stay in touch with friends, family and colleagues and take part in social events will probably change forever. CAROUSEL+ has the vision that, with the support of novel, original and imaginative combinations of Artificial Intelligence and immersive interaction technologies, people will be able to feel each other's presence, touch, and movement, even if they are physically disconnected. These new developments will help overcome isolation and loneliness and bring improvements to our health, work, and wellbeing. Moreover, they will also generate the foundations for an ecosystem of original, as yet unimagined forms of communication and expression.

Dance is a profoundly human activity. We dance when we are in love, we show we are happy with dance, sharing a dance with someone creates a deep connection. Together with language and music, dance is one of the few behaviors that occurs naturally in chil-

*e-mail: k.mitchell2@napier.ac.uk

†e-mail: carmen@grassroots-arts.eu

\footnotetext{
${ }^{1}$ http://www.CarouselDancing.org
}

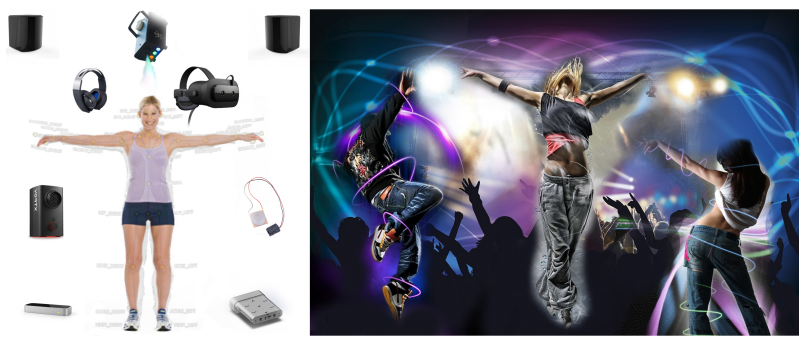

Figure 1: Imagine you feel lonely at home and wish to dance with somebody. You invite both your real friends and artificial intelligence (Al)-driven characters for a virtual house party in your living room to dance together. You can feel their touch and dancing bodies next to you and you are all enjoying this spontaneous party. You feel connected to the others and not lonely anymore. Augmented remote dance aims to overcome current challenges of learning practices hitherto restricted to the same physical space.

dren and is attested universally across world cultures [25]. Dance uniquely combines thinking, feeling, sensing, and doing. It has strong effects on physiological and psychological well-being, combining the benefits of physical exercise with heightened sensory awareness, cognitive function, creativity, inter-personal contact and emotional expression $[11,25]$. This is why in CAROUSEL+ we have chosen dance as vehicle to implement our vision.

Our studies include modern freeform dance styles in groups and in pairs, folk dances, and partner dances such as tango. We believe dance presents a special challenge due to the complex dependency between the motions, the music, the tactile contact and the dancers' feelings and sensations. The results learned from dance will open up research avenues to many other use cases in the future, from physical training to manual assembly, martial arts, companionship etc., that require collaboration and synchronization of movements in groups.

A person may feel lonely or isolated if they find themselves with nobody to contact, temporary confined, in pandemic quarantine or lockdown conditions. This person may open an interaction with a digital character and start dancing together; other real people may decide to join (figure 2). In live events an icebreaker, for example from a group animator, an inspiring dancer, musician, moderator, trainer, or companion is often needed to kick-start the interaction. We imagine that an autonomous digital character will be able to play this role in the future. This is precisely the technical CAROUSEL+ breakthrough: to create AI-driven characters who will be able to interact autonomously with a single person or a group of people in a meaningful way and the people can feel and dance with each other even if they are physically not in the same space!

Therefore, CAROUSEL+ aims to deliver a fully immersive- 


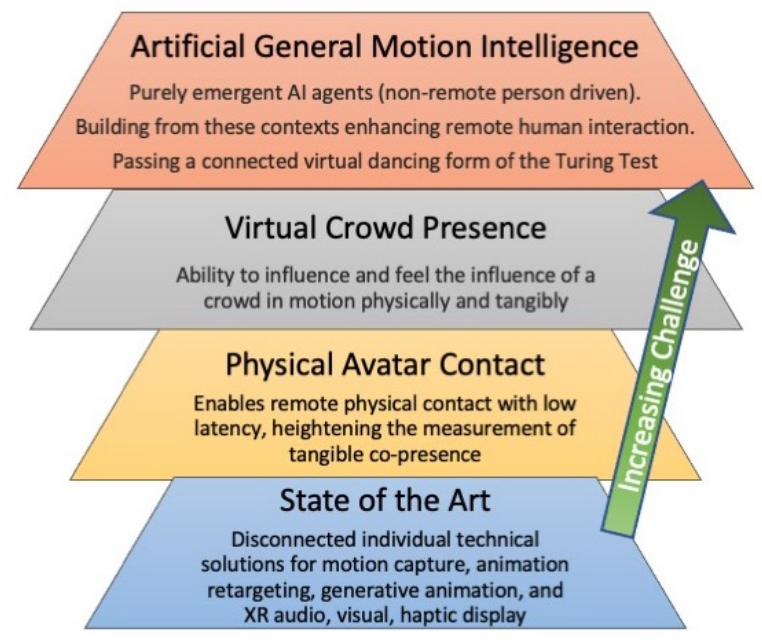

Figure 2: Levels of potential of real-world social and physical Al with autonomous digital characters.

shared performance environment through which participants may visualize their interactions with each other and AI-characters in a virtual space or in a mixed reality reconstruction of their rooms to lay the foundation for networked synchronized virtual dancing as well as other physical activities. CAROUSEL+ aims to lay the new technical and scientific foundation for social and physical real-world AI for enhanced social interaction with digital characters (Figure 2).

Many interaction and movement synthesis tasks benefit from the ability of predicting the user's movements and/or intention based on past movements and sensor data. However, for many interaction tasks, predicting poses and movement trajectories is not enough, and the user's movement needs to be understood in terms of the movement vocabularies of particular dance styles, and ergonomic and biomechanical properties such as energy expenditure and perceived effort. Furthermore, for the AI system to adapt the difficulty or to give constructive criticism on various poses or dance styles it is also necessary to evaluate the user's fatigue needs based on kinematic data alone. However, a full-body model for real-time perceived fatigue analysis and motion synthesis has yet to be developed.

\section{Interdisciplinary Co-design Methodology}

This project involves technical, scientific, social science, innovation, and artistic partners from the outset. The focus on dance is the beginning of our research path to explain our research concepts. The consortium follows the interdisciplinary co-design methodology for the development of the scenarios, use cases, requirements for the interactive formats and technologies, demonstrations, and validations. From the start of the project, the consortium partners are involving interdisciplinary co-designers in their cities, networks, and campuses, holding focus groups with artists, musicians, design students, technologists, and scientists.

In our research, we concentrate on the emergent method to study novel forms of social interaction between groups of humans and digital characters. Emergent behavior of a system cannot be described by the behavior of the components of the system and is therefore unexpected to a designer or observer. The development of new interactive technologies takes place within Research and Development aspects of computer science and technology on one side and artistic and social science on the other. These worlds are characterized by different thought processes, work techniques, strengths, and needs. Unravelling the potential of all sides in order to find unusual innovative solutions requires close interdisciplinary collaboration.

Our concept, scenarios and experiments are based on hypotheses that emergent behavior will arise from the interactions between members of a group, rather than from the behavior of the members of the group in isolation. The idea is that unforeseen, yet unknown forms of expression and communication will develop between humans and digital characters in our experiments. This should lead to the emergence of a new generation of digital characters being capable of motivated, autonomous interaction with humans.

Two things are clear about artificial intelligence (AI) and extended reality (XR). First, the technologies will be flooding the consumer, enterprise, and education markets in the coming decades. Second, researchers know little to nothing about how this novel technology will change social interaction. In CAROUSEL+ we will run user studies with different test user groups (ranging from experts to beginner dance students of all ages) to explore how group interaction will change by staging trials, experiments and live dance events with XR and AI-characters. "Experts" in this regard include dance experts, such as teachers and hobby dancers who may have not a lot of expertise in XR technologies, and XR experts, such as developers and psychologists who may not have much experience in dancing, or a combination of both expert types.

\section{Enabling Technology Objectives}

CAROUSEL+ aims to develop a complete solution for realistic augmentation and interaction of people with a never before seen level of quality and presence in the dynamic virtual environment. This is expected to have a large impact on other uses and applications of extended reality. The integration and experimental validation for the dancing experience of the proposed visual, haptic, and aural technologies providing consistent low-latency multi-modal feedback is of great research interest and challenge. Whilst hardware improves and develops, alternative combinations of devices will suit levels of sensing and display with increasing freedom of movement towards ideally fully unencumbered immersive dance in the presence of virtual dance partners.

\subsection{Audio}

Audio is a critical component to rendering an immersive feedback for remote participants to feel tele-present in a virtual shared environment and key to musical rhythmic dance. CAROUSEL+ will focus upon binaural audio reproduction over headphones/earphones, in line with industry practice for monitoring live-performance and production. By contrast, spatial audio reproduction over loudspeakers tends to require large numbers of loudspeakers, careful positioning, and calibration, and suffers limited isolation between personal sound delivered to multiple listeners in the same room $[9,28]$.

Traditionally, mixes for feedback during film/music production are channel-based whereas interactive media tend to be object-based, such as games and emerging broadcast technologies [13]. For an auditory display to sound realistically, one must include both sound sources and their reverberation with the (virtual) acoustical environment [29]. Current research is exploring methods to avoid poor localization, front-back confusions, and a lack of externalization (i.e., failing to display the sound outside of the listener's head) and context of the real environment's acoustic materials [10].

CAROUSEL+ will advance feedback methods to give an objectbased immersive experience, and will employ machine-learning methods to select appropriate features from images and anthropometric data to estimate a close match to each actor's individual headrelated impulse response (HRIR), e.g., via sparse representation [He15]. The novel contributions to audio rendering in CAROUSEL+ will be to demonstrate the use of object-based spatial audio for virtual auditory displays in synchronized networked shared experiences, 
to investigate the effect of virtual location acoustics within this representation, and to adapt the rendered scenes according to the various roles in the dance or setting. Key research questions to be addressed with respect to immersive audio experiences in CAROUSEL+ are:

- How can object-based spatial audio techniques bring benefit to a person's immersion via a virtual auditory display that is adapted to his/her anthropometric measurements and delivered binaurally?

- What influence does the impression of the acoustics of the virtual environment have on the person's immersion within that environment?

- How can auditory displays be designed to best scale to large groups and crowds?

\subsection{Visual}

CAROUSEL+ will deliver a combination of novel science (algorithms, representations) and engineering contributions to extend intelligent predictive motion sensing to real-time networked comotions. This will enable real-time immersive remote visualization of partners' virtual character performances. For addressing latency, motion prediction schemes [1] will surmount latency of body motion reconstruction among remote participants.

Although finger tracking involves capturing mainly fine scale details and motion, it is nevertheless important due to the nuances and subtleties they exhibit particularly in dance. Further, finger tracking will be leveraged by the haptic feedback system to provide the participants with unprecedented sensorimotor immersion in their connected performance and play environment

We propose to advance the state of the art using integrated camera sensors with head mounted sensors, coupled with hand-held motion sensors for open, bespoke, easily deployable, new hardware frameworks. For transmitting facial avatar expressions with greater detail than before in the first instance, we will address eyes and mouth region by extending methods of facial capture using cameras mounted inside the immersive display headset $[6,30]$. In the second instance, we will modify our solution to fuse camera sensor and immersive headset tracking information, covering the volume of each remote person, to specifically merge sensor data with as few encumbrances as possible. The aim, in each instance, is to acquire enough information to track key-features by locating cameras in the appropriate view for detailed facial, hand and body expression reconstruction. Recent promising work of the consortium on vision based facial capture image-based feature detail [20] and employs deep learning with synthesis and large 2D and 3D training sets [8].

\subsection{Haptics}

Current haptic feedback displays are not greatly suited to broad audience usage scenarios. Commercial devices, such as the CyberForce system, offer impressive force feedback capabilities but are cumbersome and adversely affect the range of motion and comfort of the wearer. Vibrotactile displays have recently gained popularity for gaming and industrial applications as they have smaller form factor and are therefore are less intrusive ${ }^{2}$. However, these solutions are not designed with a dance group in mind. Furthermore, the associated rendering methods are limited in their ability to provide rich, perceptually plausible feedback, although recent work has begun to focus on developing perceptual rendering methods for multi-grid displays creating highly immersive effects $[3,15,16,26]$.

A tangible result of CAROUSEL+ will be a sparse targeted-body haptic display, developed by consortium partners, which is capable

${ }^{2}$ Gaming vests provide real-time feedback about a player's virtual presence within a gaming environment (Tactsuit, KORFX and VYBE chair adaption) provide similar feedback for film viewing or racing game scenarios. of display a range of physical and non-physical effects to a participant, thus increasing their sense of presence in a virtual setting and providing spatiotemporal cues through touch and temperature sensing modality. The haptic actuators will utilize innovative design of vibrotactile hardware, which allows direct integration onto textiles. Hence considerably reducing any obstruction of movement important for dance. These will further provide optical tracking points for use with the sensor hardware, providing a whole-body sensorimotor solution. The haptic rendering methods will likewise leverage novel retargeting methodologies of CAROUSEL+ for providing realistic tactile stimulus to each person whose dance movements are being used to control their virtual character, mapping tangible interactions to each person's body appropriately.

\section{Social, Creative and autotelic al for Dance}

CAROUSEL+ develops technology for intelligent simulation and control of physically plausible virtual characters. Interpreting the signals from multiple people in real-time with the purpose of driving high quality animated content underpinning seamless and engaging visual experiences in the context of dancing.

\subsection{User Analysis}

CAROUSEL+ tackles the many unsolved research questions related to real-time embodied interaction: Prediction of future movements from partial sensor data, understanding of user intend in various contexts. This requires generative and predictive movement models that can be flexibly conditioned on subsets of observed variables, preferably without retraining for each possible subset. The models should also support accessibility, style transfer and adaption of multimodal feedback according to skills, strength, flexibility observations. Objectives include,

- Further building on neural generative models (e.g., StyleGAN 2 [18] and the DRMM [14]).

- Current approaches [2] in movement and dance style classification rely on a discretization of movement patterns, which narrows and specifies the number of dance styles for example. We will aim at extending such classifications to a continuous representation allowing a variety of styles.

- Increase value of feedback on posture or dance routine by incorporating models from biomechanical literature based on computational efficient torque and force analysis for kinematic fatigue measurements $[21,32]$.

- Incorporating current emotion recognition methods for explainable and valuable feedback.

A challenge that needs to be addressed is the adaption of these models to different users. To address this adaptation, next to various body measurements described in literature, CAROUSEL+ aims to develop the first full-body perceived fatigue analysis that can also be used for efficient motion synthesis with the optimization of rewards. For this we hope to combine recent movement synthesis approaches based on joint actuation torques [17] and our recently developed cumulative fatigue model for reinforcement learning (RL) [7], which uses cumulative fatigue to synthesize arm movements.

Furthermore, the machine learning models providing the estimates need to be explainable, for an AI movement partner such as a virtual dance teacher to be able to provide effective corrective feedback ("this is how you should correct your movement") instead of just summative feedback ("this movement is incorrect"). Explainability is an active topic in machine learning, mostly pursued in the domain of medical image analysis; we will review the literature, apply, and extend the most promising approaches to movement. In addition to movement classification and analysis there has already 
been work done on emotion recognition from image data specifically for VR, handling the partially occluded face due to the head mounted display [33]. If other sensors for face muscle activation [19,22] or EEG [23] are available, they can also be used for emotion detection.

\subsection{Character Animation for Dancing}

CAROUSEL+ will investigate how to compute synchronized dance motions combining physical simulation that incorporates captured sensing performance data with anatomical knowledge. The algorithms will further ensure that the dance can be augmented and enhanced such that they conform to group constraints while not encumbering group members in an undesired manner. For the simulation of the dance use cases, we want to develop a framework for dancing motions that can adapt to input signals derived from real user motion such as rhythm and interact with the environment. To our knowledge no prior AI model exists that can interact and dance with humans, handle all kind of unpredictable behavior, give feedback and simultaneously come up with new creative movements and autotelic behavior in real-time. While Granados et al. [12] have developed a robot dance teacher, which provides haptic feedback to the user, it is not able to come up with its own new dance moves, nor analyse the user's intention and behavior.

We plan to apply reinforcement learning of physics-based animation controllers. Increasing complexity in dance motions during training can be addressed by curriculum learning. Dancing requires a lot of creative and autotelic behavior to emerge that is intrinsically motivated. In reinforcement learning such motivation can be modelled by intrinsic rewards. In artificial agents, intrinsic motivation resembles curiosity and exploration. In reinforcement learning such motivation can be modelled by intrinsic rewards [4,27]. Research $[5,24]$ suggests that such intrinsic rewards correspond to the need for novel stimuli, i.e. rewarding actions that yield novel or unpredicted observations.

To satisfy dancers quest for creativity, we want to be able to "teach" custom motion to the characters by copying emergent behavior directly from the online dancers. This will lead to the development of spontaneous dance improvisational skills.

The rigid body simulations only approximate the complex musculoskeletal system of a human. Therefore, unrealistic motions can be produced by a simulation. Increasing the complexity of the model will result in a loss of performance, therefore we intend to apply fatigue models to simulate the effect of muscle systems.

To avoid having to retrain each individual character separately and thus saving time and space, we plan to introduce reusable controllers for different AI-characters.

\section{OUtLOOK}

The innovation perspective of this new branch "Real-World Social and Physical AI" is tremendous as it can be applied to many applications in many areas, for physically interacting in a meaningful social way. In particular digital characters in the future could act as physical trainers, dancers, entertainers, actors, coworkers, health assistants, guides, educators, spectators, physical therapists and companions. Beyond social, entertainment, health and educational applications, CAROUSEL + learning and understanding of body language and group dynamics could be deployed in many other areas, including security, peace-making, emergency handling and autonomous driving.

In our networked dance learning framework we can foresee taking advantage of regular rhythmic patterns, as exemplified by video games such as Just Dance, Beat Saber and Dance Central VR, but extended in guidance of cooperative, tactile and responsive motions with partners, going beyond learning by copying, but learning by leading and being led with anticipation and spontaneous response of the others' moves. Further, live motion analysis in a body tracked XR session provides a data stream that can be adapted to deliver encouraging corrections, enhancements and amplification of dance sequences.

\section{ACKNOWLEDGMENTS}

CAROUSEL + is research and innovation project number 101017779, funded under the European Horizon 2020 FET Proactive program.

\section{REFERENCES}

[1] S. Andrews, I. Huerta, T. Komura, L. Sigal, and K. Mitchell. Real-time Physics-based Motion Capture with Sparse Sensors. In Proceedings of the 13th European Conference on Visual Media Production (CVMP 2016), CVMP 2016, pp. 1-10. Association for Computing Machinery, New York, NY, USA, Dec. 2016. doi: 10.1145/2998559.2998564

[2] A. Aristidou, E. Stavrakis, M. Papaefthimiou, G. Papagiannakis, and Y. Chrysanthou. Style-based motion analysis for dance composition. The Visual Computer, 34(12):1725-1737, Dec. 2018. doi: 10.1007/ s00371-017-1452-z

[3] P. Bach-Y-Rita, C. C. Collins, F. A. Saunders, B. White, and L. Scadden. Vision Substitution by Tactile Image Projection. Nature, 221(5184):963-964, Mar. 1969. Number: 5184 Publisher: Nature Publishing Group. doi: 10.1038/221963a0

[4] G. Baldassarre and M. Mirolli. Intrinsically Motivated Learning Systems: An Overview. In G. Baldassarre and M. Mirolli, eds., Intrinsically Motivated Learning in Natural and Artificial Systems, pp. 1-14. Springer, Berlin, Heidelberg, 2013. doi: 10.1007/978-3-642-32375-1_1

[5] M. Bellemare, S. Srinivasan, G. Ostrovski, T. Schaul, D. Saxton, and R. Munos. Unifying Count-Based Exploration and Intrinsic Motivation. Advances in Neural Information Processing Systems, 29:1471-1479, 2016.

[6] C. J. D. S. Brito and K. Mitchell. Recycling a Landmark Dataset for Real-time Facial Capture and Animation with Low Cost HMD Integrated Cameras. In The 17th International Conference on VirtualReality Continuum and its Applications in Industry, VRCAI '19, pp. 1-10. Association for Computing Machinery, New York, NY, USA, Nov. 2019. doi: 10.1145/3359997.3365690

[7] N. Cheema, L. A. Frey-Law, K. Naderi, J. Lehtinen, P. Slusallek, and P. Hämäläinen. Predicting Mid-Air Interaction Movements and Fatigue Using Deep Reinforcement Learning. In Proceedings of the 2020 CHI Conference on Human Factors in Computing Systems, pp. 1-13. Association for Computing Machinery, New York, NY, USA, Apr. 2020.

[8] A. Chen, Z. Chen, G. Zhang, K. Mitchell, and J. Yu. Photo-Realistic Facial Details Synthesis From Single Image. pp. 9428-9438. IEEE Computer Society, Oct. 2019. doi: 10.1109/ICCV.2019.00952

[9] P. Coleman, P. J. B. Jackson, M. Olik, M. Møller, M. Olsen, and J. Abildgaard Pedersen. Acoustic contrast, planarity and robustness of sound zone methods using a circular loudspeaker array. The Journal of the Acoustical Society of America, 135(4):1929-1940, Apr. 2014. Publisher: Acoustical Society of America. doi: 10.1121/1.4866442

[10] D. W. Crawford, A. Samdahl, J. Voris, I. B. Kadar, and K. Mitchell. Augmented reality (AR) audio with position and action triggered virtual sound effects, Sept. 2014.

[11] R. Elliott. The Use of Dance in Child Psychiatry. Clinical Child Psychology and Psychiatry, 3(2):251-265, Apr. 1998. Publisher: SAGE Publications Ltd. doi: 10.1177/1359104598032008

[12] D. F. P. Granados, B. A. Yamamoto, H. Kamide, J. Kinugawa, and K. Kosuge. Dance Teaching by a Robot: Combining Cognitive and Physical Human-Robot Interaction for Supporting the Skill Learning Process. IEEE Robotics and Automation Letters, 2(3):1452-1459, July 2017. Publisher: Institute of Electrical and Electronics Engineers Inc. doi: 10.1109/LRA.2017.2671428

[13] J. Herre, J. Hilpert, A. Kuntz, and J. Plogsties. MPEG-H Audio-The New Standard for Universal Spatial/3D Audio Coding. Journal of the Audio Engineering Society, 62(12):821-830, Jan. 2015. Publisher: Audio Engineering Society.

[14] P. Hämäläinen, T. Saloheimo, and A. Solin. Deep Residual Mixture Models. arXiv e-prints, 2006:arXiv:2006.12063, June 2020. 
[15] A. Israr and I. Poupyrev. Control space of apparent haptic motion. In 2011 IEEE World Haptics Conference, pp. 457-462, June 2011. doi: 10.1109/WHC.2011.5945529

[16] A. Israr, I. Poupyrev, C. Ioffreda, J. Cox, N. Gouveia, H. Bowles, A. Brakis, B. Knight, K. Mitchell, and T. Williams. Surround Haptics: sending shivers down your spine. In ACM SIGGRAPH 2011 Emerging Technologies, SIGGRAPH '11, p. 1. Association for Computing Machinery, New York, NY, USA, Aug. 2011. doi: 10.1145/2048259. 2048273

[17] Y. Jiang, T. Van Wouwe, F. De Groote, and C. K. Liu. Synthesis of biologically realistic human motion using joint torque actuation. ACM Transactions on Graphics, 38(4):72:1-72:12, July 2019. doi: 10. $1145 / 3306346.3322966$

[18] T. Karras, S. Laine, M. Aittala, J. Hellsten, J. Lehtinen, and T. Aila. Analyzing and Improving the Image Quality of StyleGAN. pp. 8110$8119,2020$.

[19] H. Li, L. Trutoiu, K. Olszewski, L. Wei, T. Trutna, P.-L. Hsieh, A. Nicholls, and C. Ma. Facial performance sensing head-mounted display. ACM Transactions on Graphics, 34(4):47:1-47:9, July 2015 doi: $10.1145 / 2766939$

[20] Y. Li, L. Ma, H. Fan, and K. Mitchell. Feature-preserving detailed 3D face reconstruction from a single image. In Proceedings of the 15th ACM SIGGRAPH European Conference on Visual Media Production, CVMP '18, pp. 1-9. Association for Computing Machinery, New York, NY, USA, Dec. 2018. doi: 10.1145/3278471.3278473

[21] J. M. Looft, N. Herkert, and L. Frey-Law. Modification of a threecompartment muscle fatigue model to predict peak torque decline during intermittent tasks. Journal of Biomechanics, 77:16-25, Aug. 2018. doi: 10.1016/j.jbiomech.2018.06.005

[22] I. Mavridou, J. T. McGhee, M. Hamedi, M. Fatoorechi, A. Cleal, E. Balaguer-Ballester, E. Seiss, G. Cox, and C. Nduka. FACETEQ: A novel platform for measuring emotion in VR. In Proceedings of the Virtual Reality International Conference - Laval Virtual 2017, VRIC '17, pp. 1-3. Association for Computing Machinery, New York, NY, USA, Mar. 2017. doi: 10.1145/3110292.3110302

[23] J. Nam, H. Chung, Y. a. Seong, and H. Lee. A New Terrain in HCI: Emotion Recognition Interface using Biometric Data for an Immersive VR Experience. arXiv e-prints, 1912:arXiv:1912.01177, Dec. 2019.

[24] D. Pathak, P. Agrawal, A. A. Efros, and T. Darrell. Curiosity-driven exploration by self-supervised prediction. In Proceedings of the 34th International Conference on Machine Learning - Volume 70, ICML'17, pp. 2778-2787. JMLR.org, Sydney, NSW, Australia, Aug. 2017.

[25] A. Pickard and D. Risner. Dance, health and wellbeing special issue. Research in Dance Education, 21(2):225-227, 2020.

[26] P. Preechayasomboon, A. Israr, and M. Samad. Chasm: A Screw Based Expressive Compact Haptic Actuator. In Proceedings of the 2020 CHI Conference on Human Factors in Computing Systems, pp. 1-13. Association for Computing Machinery, New York, NY, USA, Apr. 2020.

[27] S. Roohi, J. Takatalo, C. Guckelsberger, and P. Hämäläinen. Review of Intrinsic Motivation in Simulation-based Game Testing. In Proceedings of the 2018 CHI Conference on Human Factors in Computing Systems, CHI '18, pp. 1-13. Association for Computing Machinery, New York, NY, USA, Apr. 2018. doi: 10.1145/3173574.3173921

[28] S. Spors, H. Wierstorf, A. Raake, F. Melchior, M. Frank, and F. Zotter. Spatial Sound With Loudspeakers and Its Perception: A Review of the Current State. Proceedings of the IEEE, 101(9):1920-1938, Sept. 2013. Conference Name: Proceedings of the IEEE. doi: 10.1109/JPROC. 2013.2264784

[29] K. Sunder, J. HE, E. L. Tan, and W. Gan. Natural Sound Rendering for Headphones: Integration of signal processing techniques. IEEE Signal Processing Magazine, 32(2):100-113, Mar. 2015. Conference Name: IEEE Signal Processing Magazine. doi: 10.1109/MSP.2014.2372062

[30] S.-E. Wei, J. Saragih, T. Simon, A. W. Harley, S. Lombardi, M. Perdoch, A. Hypes, D. Wang, H. Badino, and Y. Sheikh. VR facial animation via multiview image translation. ACM Transactions on Graphics, 38(4):67:1-67:16, July 2019. doi: 10.1145/3306346.3323030

[31] N. Weinstein and T.-V. Nguyen. Motivation and preference in isolation: a test of their different influences on responses to self-isolation during the covid-19 outbreak. Royal Society Open Science, 7(5):200458, 2020.
[32] T. Xia and L. A. Frey Law. A theoretical approach for modeling peripheral muscle fatigue and recovery. Journal of Biomechanics, 41(14):3046-3052, Oct. 2008. doi: 10.1016/j.jbiomech.2008.07.013

[33] H. Yong, J. Lee, and J. Choi. Emotion Recognition in Gamers Wearing Head-mounted Display. In 2019 IEEE Conference on Virtual Reality and $3 D$ User Interfaces (VR), pp. 1251-1252, Mar. 2019. ISSN: 26425254. doi: 10.1109/VR.2019.8797736 